\title{
Research on Variation of Reactive Power Capacity under DC Bias of Transformers
}

\author{
Ying-Fa HE ${ }^{1}$, Hang ZHAO ${ }^{1 a^{*}}$, Chang LIU ${ }^{1}$, Xuan $\mathrm{ZHOU}^{1}$, Cao-Wei WU ${ }^{1}$, Zhi- \\ Xun WANG ${ }^{1}$ and Ying ZHENG ${ }^{2, b}$
}

\author{
${ }^{1}$ State Key Laboratory of Advanced Electromagnetic Engineering and Technology, Huazhong \\ University of Science and Technology, Wuhan, Hubei, China \\ ${ }^{2}$ Panyu Power Supply Bureau of Guangzhou Power Supply Bureau Co., Ltd \\ Guangzhou, Guangdong, China \\ *a1040937139@qq.com b25447770@qq.com
}

\begin{abstract}
Keywords: HVDC, DC bias, Transformer, Excitation current, Reactive power compensation capacity.
\end{abstract}

\begin{abstract}
When the high voltage direct current (HVDC) transmission lines run in monopole grounding operation or bipolar asymmetric operation, most of the DC current will flow the loop consisted of the grounding electrode and AC lines between transformers. This current increases the reactive power consumption of the transformer and threats the normal operation of the power grid. To solve this problem, this paper focuses on the increasing of excitation current when the transformer works on DC magnetic bias, and a simulation model of substation system under DC bias condition is established based on PSCAD. Quantitative analysis of the variation of the excitation current and the condition of reactive power consumption of transformers under different biasing current injection is carried out. The result shows that with the increase of the DC biasing current, the transformer excitation current distortion and reactive power loss increases sharply, which is important to the research on DC current and the reactive power consumption of the transformer under different bias current injections.
\end{abstract}

\section{Introduction}

When the high voltage direct current (HVDC) system operates in ground return and bipolar imbalance mode, DC current with high amplitude will penetrate into the earth via the grounding electrode, and such powerful DC current into the ground will form a constant DC current field in the soil near the grounding electrode. At this point, the potential of the transformer around the grounding electrode rises up. While there is potential difference between the two grounding networks of the substation, DC current will flow through the neutral-grounded transformers to the winding of the transformer, causing DC bias and saturation of transformer core, and leading to a series of negative phenomenon, such as vibration intensifying and the increase of transformers noise and harmonic. Not only does DC bias threat the transformer itself and the operation safety of the electric network, but also makes noise pollution to the surrounding environment, seriously disturbs the production and living of residents in the vicinity[1-3].

At present, research in this area at home and abroad mainly focus on the analysis of operating characteristics and isolation measures of the transformer after DC current invades the neutral point. Some domestic scholars and research units have already done some research on DC bias's effects on reactive power compensation equipment. Reference [4] gives out the conclusion that there is the linear relationship between transformer reactive power consumption and the windings GIC. Reference [5] gives out the coping approach to enhance the series reactance value and avoid 4 times of harmonic amplification between the capacitors and strengthen the ability to endure DC bias. Reference [6] draws the correlation between DC biasing current and breakdown voltage of the system. Current research in China and abroad on characteristics of reactive power under DC biasing remains to go further, and DC bias's effects on the capacity of reactive power compensation equipment is still not covered. 
Therefore, aiming at the insufficiency of existing research, and according to magnetic delay theory, this paper analyzes the mechanism of DC bias's effects on excitation current, reveals the quantitative relation between DC bias and capacity occupancy of reactive compensation equipment, and provides theoretical support for the studies on short circuit voltage support capability under DC bias.

\section{Mechanism of DC Bias's Effects on Excitation Current}

Under normal circumstances, small amount of excitation current can produce the rated working magnetic flux, if a certain amount of DC current (10 20A) invades the transformer, the DC magnetic flux it produced will be much larger than the nominal magnetic flux, which will make the main magnetic offset to one side, and cause the high degree of saturation of the iron core and serious distortion of excitation current[7].

\section{Mechanism of DC Bias's Effect on Excitation Current Distortion}

According to the research in home and abroad, DC biasing effects on different types of transformer is different. Under DC biasing, Fourier analysis of excitation current of three different types of transformer is shown in Figure 1:
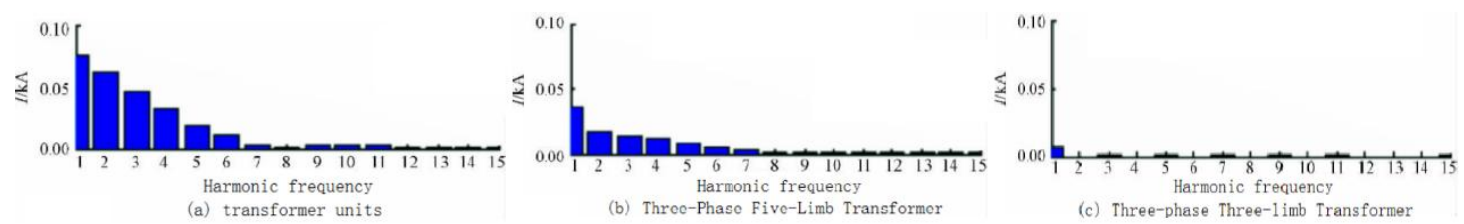

Fig. 1 DC bias degree of different types of transformers under same biasing current

Figure 1 shows that the harmonic proportion of three-phase transformer is the highest in the group, and it is most sensitive to DC bias. When the transformer operates normal, there will be no even harmonics, so it can be learned, the appearing of even harmonics is a particular phenomenon produced under DC biasing condition. The even harmonics can be used as a judgment on whether DC bias happens in the monitoring device.

In addition, the transformer is often used as main transformer in substation transformer of large capacity, and three-phase transformer above-mentioned is made up of three single-phase transformers, so this paper will mainly analyze the characteristic of single-phase transformer.

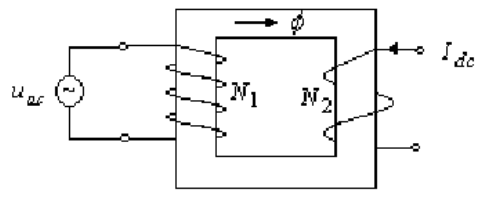

Fig. 2 Schematic diagram of single phase transformer

Figure 2 shows the equivalent figure of single-phase transformers, the voltage of primary side is:

$$
U_{a c}=\sqrt{2} U_{1} \cos \omega t \approx N_{1} \frac{d \Phi}{d t}
$$

There is a DC current ${ }^{I}{ }_{d c}$ in the secondary side winding, and assuming that all flux core are closed, denoted $\Phi$, and magnetic flux leakage effect be considered by a leakage magnetic coefficient K, DC bias in the system causes the appearing of DC magnetic flux in the transformer, that is: 


$$
\Phi=\Phi_{0}+\frac{\sqrt{2} U_{1}}{\omega N_{1}} \sin \omega t
$$

Research shows that, except for the correspondence between secondary side DC current ${ }^{I} d c$ and magnetic flux $\Phi_{0}$ caused by it, due to the effect of AC magnetic flux, there should be the difference coefficient $^{a_{0}(m)}$ between them[7], and:

$$
\Phi_{0}=\frac{\mathrm{A}}{K y} s h^{-1}\left(\frac{N_{2} I_{d c}}{l x a_{0}(m)}\right) ; \quad a_{0}(m)=\frac{\omega}{2 \pi} \int_{0}^{T} \operatorname{ch}\left(K \frac{y}{A} \Phi_{m} \sin \omega t\right) d t
$$

In the type, $\mathrm{A}$ is equivalent to the cross-sectional area of the iron-core, 1 is the length of magnetic path of the core, $\mathrm{x}$ and $\mathrm{y}$ are parameters associated with the core magnetization curve.

Under DC bias, when density of core magnetic flux is in the unsaturated zone of magnetization curve, there should be:

$$
a_{0}(m)=\frac{\omega}{2 \pi} \int_{0}^{T} 1 d t=1
$$

Because the transformer secondary side DC current ${ }^{I}{ }_{d c}$ is in linear relationship with DC magnetic flux caused by DC bias, as the current ${ }^{i_{1}}$ shown in Figure 3. At this point, the excitation current distortion does not occur.

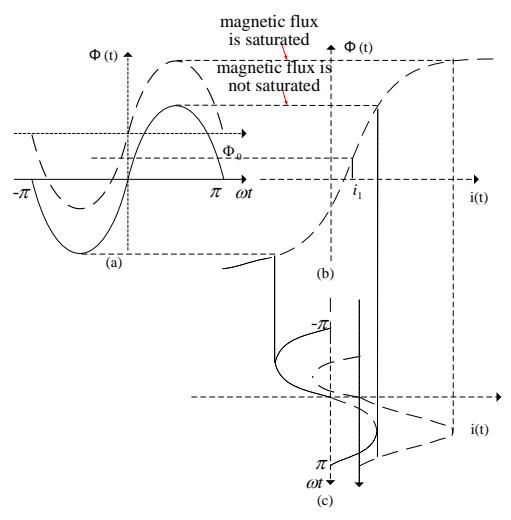

Fig. 3 Schematic diagram of DC bias

When the average magnetic flux density of iron core is in the saturated zone of the magnetization curve of the material, there should be:

$$
a_{0}(m)=\frac{\omega}{2 \pi} \int_{0}^{T} 1+\frac{\left(K \frac{y}{A} \Phi_{m}\right)^{2} \sin ^{2} \omega t}{2 !} d t=1+\frac{B_{m}^{2} y^{2}}{4} \quad ; \quad B_{m}=K \Phi / A
$$

At this point, current waveforms appear half saturation, average current is $1+\frac{B_{m}^{2} y^{2}}{4}$ times greater than current ${ }^{i_{1}}$ corresponds to DC magnetic flux on the magnetization characteristic curve. Typically, $\mathrm{y}$ values greater than 1, so there will be a degree of distortion of the excitation current waveforms.

When core magnetic flux density is in the segment of high saturation on magnetization curve:

$$
a_{0}(m)=\frac{\omega}{2 \pi} \int_{0}^{T} 1+\frac{\left(K \frac{y}{A} \Phi_{m}\right)^{4} \sin ^{4} \omega t}{4 !} d t=\frac{7 B_{m}{ }^{4} y^{4}}{192}
$$


At this time, the flux density of the core rises further, average current will be dozens of times or even hundreds of times greater than the current corresponding to the average magnetic flux. Excitation current waveform will have a more serious distortion. As the magnetization curve of the material appears nonlinear at high saturation, the linear relationship between DC magnetic flux and average excitation current no longer exists.

\section{Simulation of the Cases}

Based on PSCAD, an electromagnetic transient simulation software, this paper establishes the model of main transformer of a $500 \mathrm{kV}$ substation which contains a DC bias source[8], as shown in Figure 4.

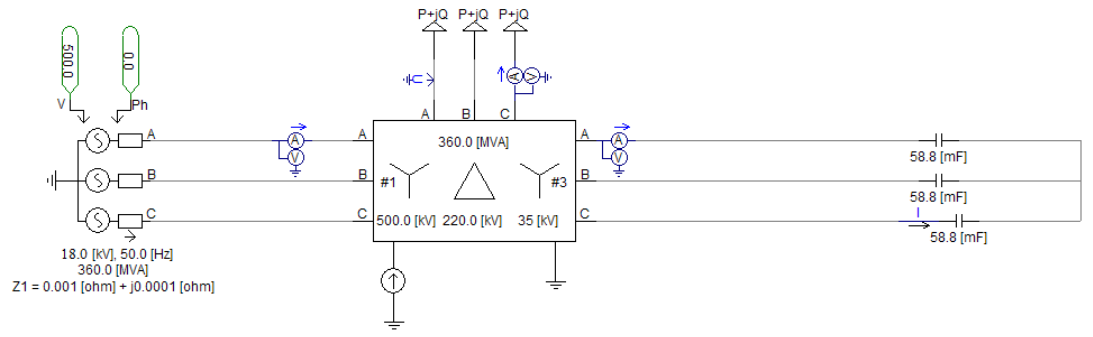

Fig. 4 Simulation model of reactive power occupation based on PSCAD

This model applies the $360 \mathrm{MVA} / 18 \mathrm{kV} / 50 \mathrm{~Hz}$ synchronous motor as the source, increases the voltage to $500 \mathrm{kV}$ through the transformer to deliver power. There are $360 \mathrm{MVA} / 500 \mathrm{kV} / 220 \mathrm{kV} / 35 \mathrm{kV}$ three winding transformers which use YNdy wiring at the end of the transmission line, simulates the DC biasing condition in the form of current source at the neutral point on the $500 \mathrm{kV}$ side of the transformer, and $220 \mathrm{kV}$ side takes the load of constant power, $35 \mathrm{kV}$ side is linked to reactive compensation capacitor, the size of each phase capacitor is $58.8 \mathrm{mF}$ (meet the current standard that reactive compensation equipment capacity accounts for 10\% 30\% of capacity of main transformer ruled by the power technology guide of power system voltage and reactive compensation). According to the regulation characteristics of SVC, the number of TSC (thyristor switched capacitors) used to compensate reactive power will be determined according to the voltage measured at the moment, rather than all of them, and use TCR (thyristor controlled reactor) to regulate the reactive power to match current reactive power consumption accurately. But because there is no limit on the reactive power output of the power the model apply, and found by the simulation, the capacity of the capacitor has little effect on the content of harmonic of current, in order to make the research more concise, we will use the fixed capacitors value for simulation.

Using PSCAD model built in this article to simulate on the excitation current is done. The waveform of the excitation current is shown in Figure 5.
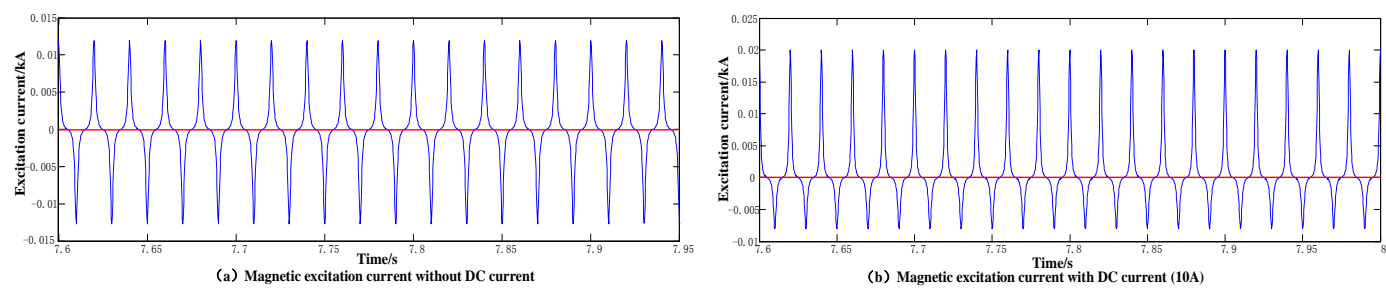

Fig. 5 Magnetic excitation current

As can be seen, without the injection of direct current, excitation current symmetric about $\mathrm{x}$ axis. Meanwhile, the amplitude of excitation current is relatively small. When the injected current is $+10 \mathrm{~A}$, compared with normal conditions, excitation current under DC biasing condition mainly has the following changes: 
1) Due to the characteristic of magnetic saturation characteristics of transformer iron cores, excitation current is no longer symmetric about the $\mathrm{x}$ axis, but significantly bias towards the positive half-axis of $y$ axis; 2) The amplitude of excitation current harmonics have grown considerably, in particular, even harmonic component appears in the excitation current;3) Amplitude of the excitation current fundamental wave increase sharply.

Meanwhile, this article makes simulation analysis under $-10 \mathrm{~A}$ current injection, the result is similar with the situation above, and we will not make further discussion here.

\section{Mechanism Analysis of DC Bias's Effects on the Increase of Reactive Power Consumption}

Based on the foregoing analysis, under DC biasing conditions, the total magnetic flux of the iron core of the transformer is superposed by DC magnetic flux and AC magnetic flux, and this will lead to the bias of the waveforms of excitation current in a particular direction, which depends on whether the injection current (the component of DC injection) is positive or negative. At this time there are three main types of important frequency components of the excitation current, namely DC component, harmonic component, fundamental component.

Other than 3 Harmonics, harmonics remaining can be passed through transformers. When the reactive power compensation equipment is an active device, due to the limits on the output range of the output current, harmonics will have a greater impact. And when the system is under a special working condition, such as short-circuit of transmission line, active reactive compensation equipment should output full power to maintain voltage level, however, harmonic component of current in reactive compensation equipment occupy the output current, but meanwhile cannot provide the fundamental reactive power the system need efficiently, which effects the ability of reactive compensation equipment to endure voltage. This paper studies on those existing practical engineering, regardless of the harmonic component's occupancy of reactive capacity.

Fundamental component is the main cause of the increase of reactive power consumption. Equivalent to circuit of transformer is shown in Figure 6:

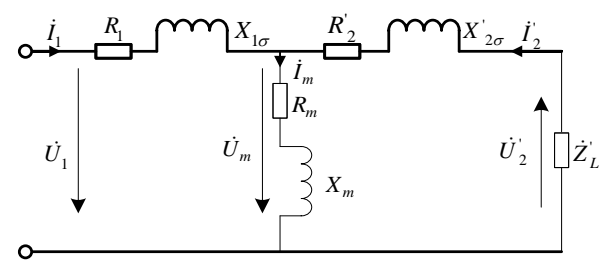

Fig. 6 Equivalent circuit diagram

As can be seen in Figure 6, the impedance of the excitation branch is not infinite. Under certain voltage, current will flow through the excitation branch, and therefore consume power.

The saturation of transformer's iron core will increase the amplitude of fundamental component of excitation current significantly, and the fundamental component and the magnetic flux of iron core are in the same phase position, lagging to the fundamental wave of the system. Therefore, the fundamental component of the excitation current can be equivalent to the reactive load of the system.

At present, there is not a clear and consistent definition of reactive power[4], the mainstream tends to define relative power as power produced by fundamental positive-sequence voltage and current, regardless of power harmonic generate. This provides an important support for the research about reactive power in this paper. If the harmonic component is contained in the definition of reactive power, and apply excitation current load model based on this definition to industrial frequency power system to analyze effect of DC injection on saturation of transformers, it will cause a lot of errors. 
Under normal conditions, the value of excitation resistance ${ }^{R_{m}}$ is very small, it's only about $1 / 10$ of a magnetizing reactance ${ }^{R_{m}}$. Therefore, the power consumed by the branch as a whole can be considered to be reactive power. Existing research directly see $Q=3 U_{m} I_{m}$ as the calculating formula of reactive power consumption in the excitation branch, however this is not justified at severe saturation of the iron core. After the iron core enter the saturation zone, the equivalent resistance and reactance will be reduced, in which reactance decrease particularly. So when the DC bias is serious, rather than simply believe that consumption is purely reactive and rely on approximate calculation of voltage and current, power measuring devices should be used to measure the consumption of reactive power, Therefore, the formula for calculating the consumption of reactive power of excitation circuit should be:

$$
\mathrm{Q}=3 U_{m} I_{m} \sin \varphi
$$

In the type, ${ }^{U_{m}}$ is the excitation branch voltages; ${ }^{I_{m}}$ is the fundamental wave current in excitation branch of transformer, ${ }^{\varphi}$ is the excitation impedance angle.

Therefore, under DC bias, when DC current makes transformer in a super saturation mood, excitation impedance will decrease sharply, and amplitude of the fundamental component of excitation current will increase significantly, which will lead to obvious increase of reactive power demand.

\section{Analysis of Examples}

Based on the simulation model built, impressive is making quantitative analysis of fundamental wave of excitation current and reactive power characteristic of transformer under different degrees of DC current injection to record DC injection and valid valve of fundamental wave of excitation current corresponding to them, as shown in Figure 7 (a):
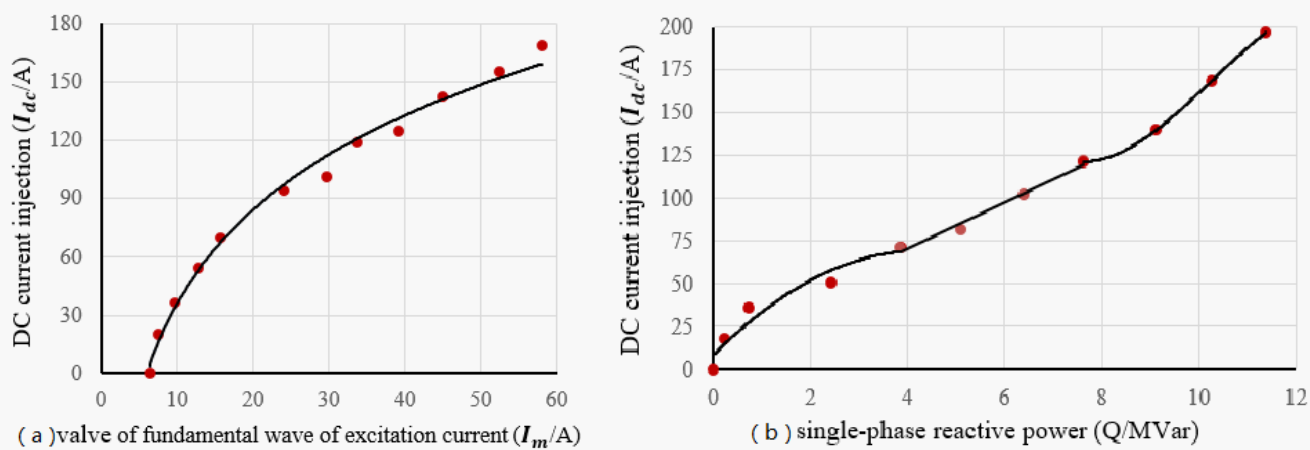

Fig. 7 Relationship between fundamental wave of magnetic excitation current, additional reactive power and DC current

According to DC current injection and valid value of excitation current fundamental wave correspond to them in Figure 7 (a), paint the point and fit the curve, it can be seen that DC current injection and valid value of excitation current fundamental wave meet index function well, so within non-saturated zone, so they can be fitted by index function, its expression type is:

$$
I_{m}=6.115 \mathrm{e}^{0.014 I_{d c}}
$$

Also, the relationship between DC biasing and increment of reactive power consumption painted by using method of polynomial fitting is shown in Figure 7 (b). As can be known from Figure 7 (b), there are three different periods of the increment of reactive power. Under DC biasing current of $0 \sim 70 \mathrm{~A}$, iron core of transformer isn't saturated, valve of equivalent reactor maintained not variable, there is the quadratic relationship between power increment and valid value of fundamental wave of 
excitation current, as the normal relationship between current and power. Under DC biasing current of 70 120A, iron core has appeared to be saturated obviously, so the power increment and excitation current increment don't meet the quadratic function anymore, but meet linear relationship approximately, and the increase of power slow down obviously. Under excitation current greater than 120A, iron core is saturated deeply, excitation impedance decreases sharply, refer to reference 6 , in spite of the sharp increase of excitation, it is still not enough to stabilize the collapse of excitation impedance, this is also a reason for the slowing down of power growth. Meanwhile, it proves the correctness of type (7) to apply the power factor as a variable to calculate reactive power loss. The relationship between biasing current and reactive increment could be expressed as:

$$
Q=\left\{\begin{array}{c}
0.008 I_{d c}{ }^{2}+0.0013 I_{d c}-0.0475 \quad I_{d c}<70 \\
0.073 I_{d c}-1.054 \quad 70<I_{d c}<120 \\
0.00001 I_{d c}{ }^{3}+0.0058 I_{d c}{ }^{2}+I_{d c}-49.58 \quad I_{d c}>120
\end{array}\right.
$$

According to the existing principles, the capacity of reactive power compensation equipment the site configured accounts for 10\% 30\% of installed capacity in the total, and in engineering 10\% is always the standard, for instance, capacity of 360MVA single-phase reactive power compensation is $12 \mathrm{MVar}$. Figure 7 (b) shows that, within the DC biasing current range which may occur in the neutral point of the transformer, the occupancy of reactive power should not be ignored compared to the capacity of the reactive power, this also shows that the DC bias can lead to loss of ability to adjust voltage of reactive compensation equipment and have a great impact on the stability of the system.

\section{Conclusions}

Based on existing research at home and abroad, this paper makes further specific analysis of the change of the excitation current and reactive power consumption under biasing current injection, here is the summary:

1) When HVDC system operates in ground return mode, DC bias will happen in main transformer of the substation near the grounding, and the biasing current will increase reactive power consumption of the transformer, occupy the capacity of reactive compensation equipment, and effect the stability of the system.

2) Using the PACAD/EMTDC software, simulation model of substation system is established based on three-phase transformer, fundamental component of excitation current and reactive power consumption increments under different DC current injection are analyzed based on the model, and according to the fitting of large amount of simulation data, the internal law of DC biasing effects on the capacity of reactive equipmentis quantified.

\section{References}

[1] Peng Liu, Qian-wen Guo, Ming Yang. Grounding method of the neutral point of DC biasing transformer [J]. High voltage technology, 2015, 41 (3): 794-799, in Chinese.

[2] Jiang-tao Quan, Zhi-cheng Xie, Ke-ji Chen, Simulation analysis of DC current distribution of the neutral point under monopole operation of UHVDC transmission system [J]. High voltage technology, 2015, 41 (3): 787-793, in Chinese.

[3] Qing-heng Chen, Hong-bin Ma, Jin-liang He. Measurement and analysis of the vibration and noise of the $500 \mathrm{kV}$ transformer caused by DC bias [J]. High voltage electrical equipment, 2009, 45 (3): 93-96, in Chinese.

[4] Hong-lan Zhang, Chen-guang Peng, Lian-guang Liu. Excitation current and its reactive power characteristics under the action of GIC [J]. Electric network and clean energy, 2010, 26 (2): 13 to 17, in Chinese. 
[5] Hai-liang Lu, Xi-shanWen, Lan Lei. The influence of dc biasing transformer on reactive power compensation capacitor [J]. High voltage technology, 2010, 4 (5): 1124-1130, in Chinese.

[6] Xiang-ning Lin, Guo-wen Yang, Han-li Weng. Analysis of the influence of the increase of reative power comsumption under dc bias on voltage of the system [J]. High voltage technology, 2016, 42 (1): 272-278, in Chinese.

[7] Ying-ying Yao. Research on DC bias of large power transformer [D]. Shenyang university of industry, 2000.

[8] Xiao-dong Zheng, Neng-ling Tai, Guang-lian Yang, et al. Modeling and simulation of UHVDC system[J]. Electric Power Automation Equipment, 2012, 32(7): 10-14, in Chinese. 